Original Research

\title{
Knee crepitus is prevalent in women with patellofemoral pain, but is not related with function, physical activity and pain
}

\section{A R T I C L E I N F O}

\section{Article history:}

Received 12 March 2018

Received in revised form

17 May 2018

Accepted 5 June 2018

\section{Keywords:}

Knee

Anterior knee pain

Physical function

Crepitus

\begin{abstract}
A B S T R A C T
Objectives: (i) To assess the reliability of knee crepitus measures, (ii) to investigate the association between knee crepitus and PFP; (iii) to investigate the relationship between knee crepitus with selfreported function, physical activity and pain.

Design: Cross-sectional.

Setting: Laboratory-based study.

Participants: 165 women with PFP and 158 pain-free women.

Main outcome measures: Knee crepitus test, anterior knee pain scale (AKPS) and self-reported worst knee pain in the last month, knee pain after 10 squats and knee pain after 10 stairs climbing.

Results: Knee crepitus clinical test presented high reliability Kappa value for PFP group was 0.860 and for pain-free group was 0.906 . There is a significantly greater proportion of those with crepitus in the PFP group than in the pain-free group $(\mathrm{OR}=4.19)$. Knee crepitus had no relationship with function $(\mathrm{rpb}=0.03 ; \mathrm{p}=0.727)$, physical activity level $(\mathrm{rpb}=0.010 ; \mathrm{p}=0.193)$, worst pain $(\mathrm{rpb}=0.11 ; \mathrm{p}=0.141)$, pain climbing stairs $(\mathrm{rpb}=0.10 ; \mathrm{p}=0.194)$ and pain squatting $(\mathrm{rpb}=0.02 ; \mathrm{p}=0.802)$.

Conclusion: Women who presents knee crepitus have 4 times greater odds to be in a group with PFP compared to those who do not. However, knee crepitus has no relationship with self-reported clinical outcomes of women with PFP.
\end{abstract}

๑) 2018 Elsevier Ltd. All rights reserved.

\section{Introduction}

Patellofemoral pain (PFP) is a common condition affecting a large proportion of young adults (Smith, Selfe, \& Rathleff, 2018), including $13 \%$ of women aged $18-35$ years (Roush \& Bay, 2012). Moreover, the prevalence of PFP in women is 2.23 times more than men (Boling et al., 2010), which makes the study of this subgroup of utmost importance. Despite presenting a high incidence, the etiology of PFP remains debated with many biomechanical alterations reported in the literature (Lankhorst, Bierma-Zeinstra, \& van Middelkoop, 2013).

Several theoretical hypotheses have been proposed in an

\footnotetext{
* Corresponding author. La Trobe University, Kingsbury Drive, Bundoora, VIC, 3086, Australia.

E-mail address: danilo110190@hotmail.com (D. de Oliveira Silva).
}

attempt to explain the pathomechanisms underlying PFP development (Lankhorst et al., 2013; Powers, Witvrouw, Davis, \& Crossley, 2017). However, what seems to be clear is the presence of some clinical signs as reported in the most recent international PFP retreat led by area experts (Crossley et al., 2016). Among commonly reported clinical signs are tenderness on patellar facet palpation, small effusion, pain on sitting, rising on sitting, squatting and climbing stairs (Crossley et al., 2016). A complete clinical examination is the keystone to assess people with PFP, as there is no gold standard clinical test to diagnose this disorder (Décary et al., 2017; Lack, Neal, De Oliveira Silva, \& Barton, 2018). Therefore, studies should properly investigate the role of all clinical signs that are supposed to be related with PFP to provide high-quality evidence-based information for clinicians managing people with PFP.

In this context, a clinical sign usually reported as a feature of people with PFP is knee crepitus (Kastelein et al., 2014; Nijs, Van 
Geel, Van Der Auwera, \& Van de Velde, 2006), which is defined as an audible grinding noise and/or palpable vibrations during joint movement (Crema et al., 2011; Schiphof et al., 2014). Knee crepitus has been reported as a clinical sign of people with PFP (Crossley et al., 2016), but no study has demonstrated if there really is an association between knee crepitus and women with PFP. Moreover, the clinical implication of knee crepitus in people with PFP was only investigated qualitatively in a patient-based perspective (Robertson, Hurley, \& Jones, 2017) and was reported to be a matter worthy of further investigation. It remains unknown if knee crepitus is related with clinical outcomes of people with PFP as poor functional capacity, physical activity level or pain during activities loading the patellofemoral joint. This information would aid clinicians and patients to better understand the matter and implication of the knee joint crepitus.

In this context, the aims of this study were: (1) to assess the reliability of knee crepitus measures, (2) to investigate the association between knee crepitus and PFP; (3) to investigate the relationship between knee crepitus and self-reported function, physical activity level, worst pain in the last month, pain climbing stairs and pain squatting.

\section{Methods}

This was a cross-sectional study reported according to STROBE guideline recommendations (von Elm et al., 2007). One-hundred sixty-five women with PFP and 158 pain-free women were recruited via advertisements at universities, gyms, public places for physical activity and posts on social media between September 2015 and April 2017. The study was approved by the Local Ethics Committee. Each participant gave written informed consent prior to participation.

\subsection{Eligibility criteria}

The eligibility criteria were based in previous studies (Crossley et al., 2016). The diagnosis of PFP was made by a clinician ( $>$ five years of clinical experience). The inclusion criteria were (1) anterior knee pain when performing at least two of the following activities: sitting for prolonged time, squatting, kneeling, running, ascending stairs, descending stairs, jumping, or landing; (2) insidious onset symptoms lasting at least 4 months; and (3) the worst pain level in the previous month corresponding to at least $3 \mathrm{~cm}$ in the visual analogue pain scale (VAS). Participants need to present all 3 criteria to be included in the PFP group. In order to be included in the painfree group participants could not present any signs or symptoms of PFP or other musculoskeletal condition. Exclusion criteria, assessed by a physiotherapist, for both PFP and pain-free groups were as follows: (1) history of surgery in any lower limb joint, (2) history of patellar subluxation or clinical evidence of meniscal injury or ligament instability, or joint effusion, (3) symptomatic osteoarthritis in any lower limb joint, (4) patellar tendon pathology, or (5) referred pain coming from the lumbar spine, hips, ankles or feet.

\subsection{Instruments}

After the screening process, all participants completed questionnaires related to physical activity and knee-related function. The first questionnaire was the self-administered International Physical Activity Questionnaire - long form (IPAQ), a valid and reliable instrument for reporting physical activity levels (Craig et al., 2003). The physical activity was determined by the total amount of physical activity in the previous week involving the lower limbs and calculated according to previous studies (Briani, Pazzinatto, Silva, \& Azevedo, 2017; Craig et al., 2003). All participants were asked to rate their knee pain by putting a mark along a $10-\mathrm{cm}$ linear VAS indicating the worst knee pain in the last month, with 0 indicating no pain and 10 the worst pain possible. VAS has been validated and it is reliable for assessing people with PFP (Crossley, Bennell, Cowan, \& Green, 2004). The last instrument applied was the anterior knee pain scale (AKPS). The AKPS is a 13item questionnaire that evaluates subjective symptoms and functional limitations associated with PFP (Kujala et al., 1993). The questionnaire score ranges from 0 to 100 , with the maximum total score of 100 indicating no disability. This tool has been validated for people with PFP and has been reported to demonstrate high test-retest reliability (Crossley et al., 2004).

\subsection{Procedures}

Knee crepitus was assessed for each participant's symptomatic limb (unilateral symptoms) or most symptomatic limb (bilateral symptoms). For the pain-free group, the dominant limb was evaluated for crepitus. For the knee crepitus assessment, the evaluator placed the palm of the hand over the patella to detect the presence of a grinding sensation during active knee flexion-extension movement (two squats until $90^{\circ}$ of knee flexion) (Souza, 1997). The test was considered positive for knee crepitus when a grinding, crackling or crunching sensation during knee extension or flexion was detected (Schiphof et al., 2014; Souza, 1997). However, just one or two clicks or pops was not considered crepitus. The rater was blind concerning pain status.

In order to assess inter-rater reliability of this knee crepitus measure, another trained rater (rater 2), with similar clinical experience, performed the same test in 85 participants of each group. The test was performed in the same conditions, 2 min after rater 1 . Raters were blinded to each other's assessments and had no contact before or after the test. The training of the rater consisted in the assessment of 10 participants under the supervision of an experienced clinician (>five years of clinical experience) and more 10 participants alone with the experienced clinician cross-checking the outcome just after the trainee assessment.

Participants then performed repeated squats and stair climbing, in a randomized order (flipping a coin), in order to assess participant's knee pain while performing patellofemoral joint loading activities. For the squatting task, participants were instructed to perform ten double leg squats continuously from a neutral position (approximately $0^{\circ}$ of knee flexion) to a depth of approximately $90^{\circ}$ of knee flexion while maintaining heel contact with the floor (Wallace, Salem, Salinas, \& Powers, 2002). A metronome was used at 60 beats/minute to standardize the cadence of the squats among participants (Wallace et al., 2002). After the squatting task, participants rated their knee pain on VAS. For the stair climbing task, the participants performed 10 continuously repetitions of stair climbing on a 7-step staircase described in a previous study (De Oliveira Silva et al., 2016). A metronome was used at 96 steps/ minute to standardize the cadence of stair climbing (Pazzinatto et al., 2016). After the stair climbing task, the participants rated their knee pain on VAS. Participants were given a 10-min rest between tasks to avoid pain summation and limit neuromuscular fatigue. In addition, participants were asked to perform the next task only when they feel completely recovered from the previous task.

\subsection{Statistical analysis}

Prior to statistical analysis, all continuous variables were assessed for normality and found to be normally distributed based on obtainment of $\mathrm{p}>0.05$ in the Kolmogorov-Smirnov test. To analyze the inter-rater reliability of the crepitus assessment, Kappa 
coefficients $(95 \% \mathrm{CI})$ and percentage agreement were calculated for both groups. Strength of the Kappa coefficients was categorized as poor $(\kappa<0.00)$, slight $(\kappa=0.00-0.20)$, fair $(\kappa=0.21-0.40)$, moderate $(\kappa=0.41-0.60)$, substantial $(\kappa=0.61-0.80)$, or almost perfect $(\kappa=0.81-1.00)$ (Landis \& Koch, 1977). A percentage agreement greater than $75 \%$ was determined to be acceptable. Mean age, body mass index (BMI) and physical activity level were compared between groups using independent $t$ tests. Chi-square tests were used to evaluate the association between the presence of knee crepitus and the presence of PFP. For the PFP group, point-biserial correlations were used to measure the strength and direction of the relationship between knee crepitus (dichotomous variable) and self-reported function, physical activity level worst pain level in the last month, pain climbing stairs and pain squatting (continuous variables). Statistical analyses were performed using SPSS version 23 (IBM, SPSS inc., Chicago, Il). The level of significance was set $a$ priori at $\mathrm{p}>0.05$.

\section{Results}

For PFP group, the Kappa value (95\% Confidence Interval) was $0.860(0.727-0.993)$ with a percentage agreement of $95.3 \%$. The Kappa value for the pain-free group was $0.906(0.816-0.995)$ with a percentage agreement of $95.3 \%$. For both groups, the inter-rater reliability and percentage agreement of the crepitus assessment was almost perfect.

There were no significant differences $(p>0.05)$ between the groups for age, BMI or physical activity level (Table 1 ). Self-reported function (AKPS), worst pain level in the last month, pain climbing stairs and pain squatting of PFP group are reported in Table 1.

From 165 women with PFP, 112 (67.88\%) presented knee crepitus and from 158 pain-free women, 53 (33.54\%) presented knee crepitus. These findings demonstrated that a greater proportion of those with PFP have knee crepitus than those who are pain free $\left(\chi_{(1)}^{2}=38.08 ; p<0.001 ; O R=4.19\right)$ (Fig. 1). No significant relationships were found between the presence of crepitus and selfreported function $\left(r_{p b}=0.03 ; p=0.727\right)$, physical activity level $\left(\mathrm{r}_{\mathrm{pb}}=0.010 ; \mathrm{p}=0.193\right)$, worst pain level in the last month $\left(\mathrm{r}_{\mathrm{pb}}=0.11 ; \mathrm{p}=0.141\right)$, pain climbing stairs $\left(\mathrm{r}_{\mathrm{pb}}=0.10 ; \mathrm{p}=0.194\right)$ or pain squatting $\left(\mathrm{r}_{\mathrm{pb}}=0.02 ; \mathrm{p}=0.802\right)$ (Fig. 2 ).

\section{Discussion}

The role of knee crepitus in women with PFP is still poorly investigated, despite being reported as a common feature of this population (Crossley et al., 2016). The aim of this study was to investigate whether knee crepitus is associated with PFP and the clinical implications of this feature. Our findings demonstrated that, knee crepitus is present with a greater proportion in women with PFP than in pain-free women. Women who present with knee crepitus have 4 times the odds to have PFP compared to those who

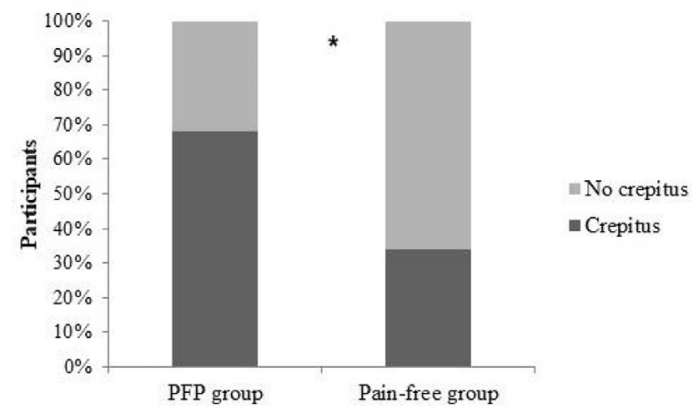

Fig. 1. Percentage of participants presenting and not presenting knee crepitus in both groups.

do not present knee crepitus. However, there seems to be no relation between crepitus and poor self-reported function, physical activity level, worst pain or pain during activities that usually provoke pain in women with PFP (e.g. stair climbing and squat).

Despite being found with a greater proportion in women with PFP, knee crepitus was also found in $33.5 \%$ of pain-free women. Findings of knee crepitus in asymptomatic population are not uncommon in the literature (McCoy, McCrea, Beverland, Kernohan, \& Mollan, 1987). For instance, McCoy et al. reported that 99\% of asymptomatic individuals present physiological patellofemoral crepitus assessed by a vibration arthrography technique. However, the authors stated that this signal was generally not seen at speeds faster than $20 \mathrm{~s}$ per cycle, and as the squat cycle was faster than $20 \mathrm{~s}$ in our study (60 beats/minute), this could explain the lower prevalence of crepitus in our asymptomatic population. In addition, the clinician's hand might not be as sensitive as the vibration arthrography technique.

To our knowledge, this is the first study reporting an association between knee crepitus and PFP. Knee crepitus has also been recently reported in people with patellofemoral osteoarthritis (Niiyama, Happle, \& Hoffmann, 2017). Knee crepitus is associated with patellofemoral joint lesions assessed by magnetic resonance imaging and self-reported pain in people with patellofemoral osteoarthritis (Riddle, Kong, \& Jiranek, 2012; Schiphof et al., 2014). A recent study (Lo et al., 2018) has also reported that in those that did not have preexisting frequent knee symptoms and did not have radiographic osteoarthritis, knee crepitus predicts symptomatic knee osteoarthritis longitudinally. The presence of knee crepitus in both disorders (PFP and knee osteoarthritis) are interesting as a recent narrative review has reported that people with PFP and patellofemoral osteoarthritis share some biomechanical features (Wyndow, Collins, Vicenzino, Tucker, \& Crossley, 2016). Our findings indicate that besides sharing biomechanical features, they may also share a clinical feature as knee crepitus. Since a larger percentage of people with PFP also have crepitus, further prospective studies investigating if crepitus is also a risk factor for PFP are

Table 1

Characteristics of the participants.

\begin{tabular}{|c|c|c|c|}
\hline & Pain-free Group $(\mathrm{n}=158)$ & PFP Group $(\mathrm{n}=165)$ & \\
\hline Variable & Mean (SD) & Mean (SD) & p-value \\
\hline Age (years) & $22.00(2.88)$ & $22.09(3.15)$ & 0.816 \\
\hline Body Mass Index & $21.70(2.77)$ & $23.43(3.77)$ & 0.101 \\
\hline Physical activity $\left(\right.$ MET $\cdot \min \cdot \mathrm{wk}^{-1}$ ) & $4329.62(485.59)$ & $4512.14(399.95)$ & 0.822 \\
\hline Self-reported function (AKPS) & $100(0.00)$ & $71.96(9.53)$ & $<0.001^{\mathrm{a}}$ \\
\hline Worst pain level in the last month (VAS) & - & $5.31(1.65)$ & - \\
\hline Pain level climbing stairs (VAS) & - & $3.20(2.48)$ & - \\
\hline Pain level squatting (VAS) & - & $3.78(2.25)$ & - \\
\hline
\end{tabular}

${ }^{a}$ Statistically significant $(\mathrm{p}<0.05)$ values. AKPS $=$ Anterior knee pain scale; VAS $=$ Visual analogue scale. 

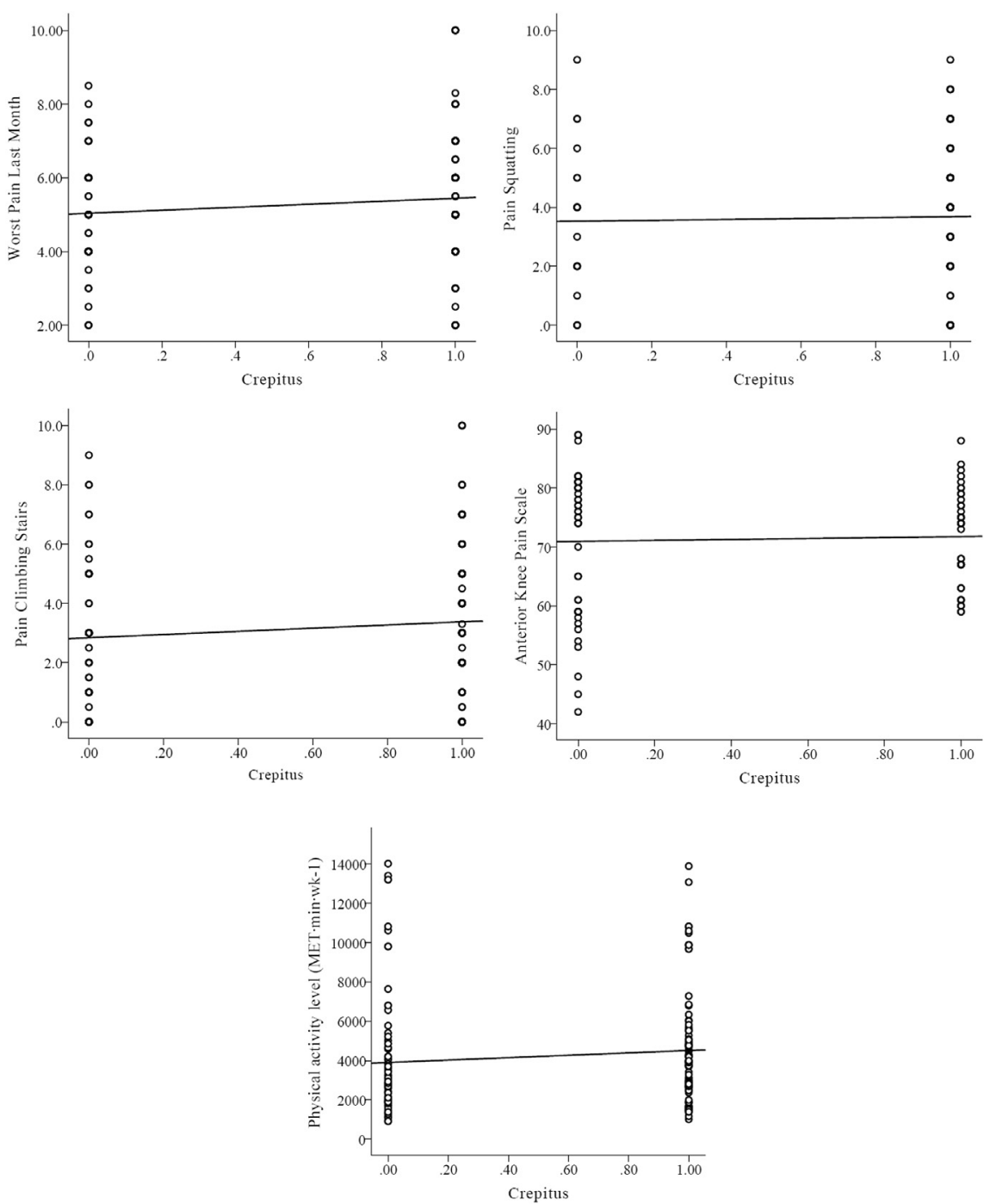

Fig. 2. Correlations between knee crepitus with self-reported pain, function and physical activity.

warranted.

While the presence of crepitus does not seem to influence function, physical activity level and pain, it does seem to influence patient perception. In a recent qualitative study in people with PFP (Robertson et al., 2017), it was reported that knee crepitus creates negative emotions, inaccurate etiological beliefs and ultimately leads to altered behaviour in patients. Many patients do not understand what the noise means in regard to their knees, assuming it is a symbol of aging, which results in avoiding physical activity, social events and even physiotherapy (Robertson et al., 2017). Those with PFP and crepitus also reported poor experiences with health professionals regarding the explanation about what knee crepitus really means for them (Robertson et al., 2017). Future studies should aim to understand both the emotional and functional effects that crepitus has in those with PFP, and if education can correct any inaccurate beliefs about crepitus.

Another comprehensive qualitative study (Smith, Moffatt, et al., 2018) reported that patients with PFP have strong desire to elucidate the cause of their knee pain. Currently, the most common perception is that physical alterations (e.g. biomechanical changes, knee crepitus, muscle weakness) are causative of PFP. In this context, our findings could be useful for health professionals to educate people with PFP on the meaning of crepitus. When educating patients about crepitus, clinicians should clarify that the sounds they hear have no relationship with pain, function or physical activities they may be involved in. This means that patients should be educated that they do not need to reduce their level of physical activity or stop physiotherapy because of the knee crepitus.

\subsection{Limitations and future directions}

This study presents some limitations that should be acknowledged. We investigated the relationship between knee crepitus during two squats with self-reported pain while squatting and negotiating stairs, however, other activities that usually cause pain in people with PFP were not investigated such as running and jumping (Crossley et al., 2016). Caution should be taken when interpreting our findings as both physical function and physical activity were self-reported, different findings could be found if these outcomes were assessed objectively. Due to the crosssectional design of our study we are unable to confirm if knee crepitus is a risk factor for the development of PFP. Further studies could prospectively investigate such relationship. Another limitation is that the sample included only younger adult women with PFP. Although this subgroup is important to study, as this 
population are the most likely to suffer from PFP, the results may not be generalizable to the entire population of people with PFP (e.g. adolescents, men and older adults). Future studies are needed to investigate the prevalence of knee crepitus in adolescents, men and older adults with PFP.

\section{Conclusion}

Our findings demonstrated that the measurement of knee crepitus is reliable and that a significantly larger proportion of those with PFP have knee crepitus than those who are pain free. Women who present with knee crepitus are at 4 times greater odds of having PFP compared to those who do not present knee crepitus. However, knee crepitus has no negative impact on function, physical activity level and pain of women with PFP.

\section{Declaration of interest}

None.

\section{Ethical approval}

The study was approved by the São Paulo State University Ethics Committee (number: 1.484.129).

\section{References}

Boling, M., Padua, D., Marshall, S., Guskiewicz, K., Pyne, S., \& Beutler, A. (2010) Gender differences in the incidence and prevalence of patellofemoral pain syndrome. Scandinavian Journal of Medicine \& Science in Sports, 20(5), 725-730. https://doi.org/10.1111/j.1600-0838.2009.00996.x.Gender.

Briani, R. V., Pazzinatto, M. F., Silva, D. O., \& Azevedo, F. M. (2017). Different pain responses to distinct levels of physical activity in women with patellofemoral pain. Brazilian Journal of Physical Therapy, 21(2), 138-143. https://doi.org/10. 1016/j.bjpt.2017.03.009.

Craig, C. L., Marshall, A. L., Sjöström, M., Bauman, A. E., Booth, M. L., Ainsworth, B. E. et al. (2003). International physical activity questionnaire: 12 -country reliability and validity. Medicine \& Science in Sports \& Exercise, 35(8), 1381-1395. https:// doi.org/10.1249/01.MSS.0000078924.61453.FB.

Crema, M. D., Guermazi, a., Sayre, E. C., Roemer, F. W., Wong, H., Thorne, a., et al. (2011). The association of magnetic resonance imaging (MRI)-detected structural pathology of the knee with crepitus in a population-based cohort with knee pain: The MoDEKO study. Osteoarthritis and Cartilage, 19(12), 1429-1432. https://doi.org/10.1016/j.joca.2011.09.003.

Crossley, K. M., Bennell, K. L., Cowan, S. M., \& Green, S. (2004). Analysis of outcome measures for persons with patellofemoral pain: Which are reliable and valid? Archives of Physical Medicine and Rehabilitation, 85(5), 815-822. https://doi.org/ 10.1016/S0003-9993(03)00613-0.

Crossley, K. M., Stefanik, J. J., Selfe, J., Collins, N. J., Davis, I. S., Powers, C. M., et al. (2016). 2016 Patellofemoral pain consensus statement from the 4th International Patellofemoral Pain Research Retreat, Manchester. Part 1: Terminology, definitions, clinical examination, natural history, patellofemoral osteoarthritis and patient-reported outcome m. British Journal of Sports Medicine, 50(14), 839-843. https://doi.org/10.1136/bjsports-2016-096384.

De Oliveira Silva, D., Magalhães, F. H., Pazzinatto, M. F., Briani, R. V., Ferreira, A. S. Aragão, F. A., et al. (2016). Contribution of altered hip, knee and foot kinematics to dynamic postural impairments in females with patellofemoral pain during stair ascent. The Knee, 23(3), 376-381. https://doi.org/10.1016/j.knee.2016.01. 014.

Décary, S., Frémont, P., Pelletier, B., Fallaha, M., Sylvain, B., Martel-Pelletier, J., et al. (April 2018). Validity of combining history elements and physical examination tests to diagnose patellofemoral pain. Archives of Physical Medicine and Rehabilitation, 99(4), 607-614. https://doi.org/10.1016/j.apmr.2017.10.014.

von Elm, E., Egger, M., Altaman, D., Pocock, S., Gotzsche, P., \& Vandenbroucke, J. P. (2007). Strengthening the reporting of observational studies in epidemiology (STROBE) statement: Guidelines for reporting observational studies. British Medical Journal, 335(10), 806-808.
Kastelein, M., Luijsterburg, P. A. J., Heintjes, E. M., van Middelkoop, M., Verhaar, J. A. N., Koes, B. W., et al. (2014). The 6-year trajectory of non-traumatic knee symptoms (including patellofemoral pain) in adolescents and young adults in general practice: A study of clinical predictors. British Journal of Sports Medicine, 49, 400-405. https://doi.org/10.1136/bjsports-2014-093557.

Kujala, U. M., Jaakkola, L. H., Koskinen, S. K., Taimela, S., Hurme, M., \& Nelimarkka, O. (1993). Scoring of patellofemoral disorders. Arthroscopy, 9(2), 159-163. Retrieved from http://www.ncbi.nlm.nih.gov/pubmed/8461073.

Lack, S., Neal, B., De Oliveira Silva, D., \& Barton, C. (July 2018). How to manage patellofemoral pain - understanding the multifactorial nature and treatment options. Physical Therapy in Sport, 32, 155-166. https://doi.org/10.1016/j.ptsp. 2018.04.010.

Landis, J. R., \& Koch, G. G. (1977). The measurement of observer agreement for categorical data. Biometrics, 33, 159-174.

Lankhorst, N. E., Bierma-Zeinstra, S. M. a, \& van Middelkoop, M. (2013). Factors associated with patellofemoral pain syndrome: A systematic review. British Journal of Sports Medicine, 47(4), 193-206. https://doi.org/10.1136/bjsports2011-090369.

Lo, G. H., Strayhorn, M. T., Driban, J. B., Price, L. L., Eaton, C. B., \& Mcalindon, T. E. (2018). Subjective crepitus as a risk factor for incident symptomatic knee Osteoarthritis: Data from the osteoarthritis initiative. Arthritis Care \& Research, 70(1), 53-60. https://doi.org/10.1002/acr.23246.

McCoy, G., McCrea, J. D., Beverland, D. E., Kernohan, W. G., \& Mollan, R. A. B. (1987). Vibration arthrography as a diagnostic aid in diseases of the knee. The Journal of Bone and Joint Surgery, 69(2), 288-293. https://doi.org/10.1016/0268-0033(87) 90023-4.

Niiyama, S., Happle, R., \& Hoffmann, R. (January 2018). Diagnostic performance of clinical examination measures and pain presentation to identify patellofemoral joint osteoarthritis. Arthritis Care \& Research, 70(1), 157-161. https://doi.org/10. 1002 /acr.

Nijs, J., Van Geel, C., Van Der Auwera, C., \& Van de Velde, B. (2006). Diagnostic value of five clinical tests in patellofemoral pain syndrome. Manual Therapy, 11(1), 69-77. https://doi.org/10.1016/j.math.2005.04.002.

Pazzinatto, M. F., de Oliveira Silva, D., Barton, C., Rathleff, M. S., Briani, R. V., \& de Azevedo, F. M. (2016). Female adults with patellofemoral pain are characterized by widespread hyperalgesia, which is not affected immediately by patellofemoral joint loading. Pain Medicine, 17(10), 1953-1961. https://doi.org/10.1093/ $\mathrm{pm} / \mathrm{pnw} 068$.

Powers, C. M., Witvrouw, E., Davis, I. S., \& Crossley, K. M. (2017). Evidence-based framework for a pathomechanical model of patellofemoral pain: 2017 patellofemoral pain consensus statement from the 4th international patellofemoral pain research retreat, part 3. Manchester, UK: British Journal of Sports Medicine. bjsports-2017-098717 https://doi.org/10.1136/bjsports-2017-098717.

Riddle, D. L., Kong, X., \& Jiranek, W. a. (2012). Factors associated with rapid progression to knee arthroplasty: Complete analysis of three-year data from the osteoarthritis initiative. Joint Bone Spine, 79(3), 298-303. https://doi.org/10. 1016/j.jbspin.2011.05.005.

Robertson, C. J., Hurley, M., \& Jones, F. (2017). People's beliefs about the meaning of crepitus in patellofemoral pain and the impact of these beliefs on their behaviour: A qualitative study. Musculoskeletal Science and Practice, 28, 59-64. https://doi.org/10.1016/j.msksp.2017.01.012.

Roush, J., \& Bay, R. C. (2012). Prevalence of anterior knee pain in 18-35 year-old females. International Journal of Sports Physical Therapy, 7(4), 396-401. https://doi.org/10.1016/j.jsams.2011.05.004

Schiphof, D., Van Middelkoop, M., De Klerk, B. M., Oei, E. H. G., Hofman, a., Koes, B. W., et al. (2014). Crepitus is a first indication of patellofemoral osteoarthritis (and not of tibiofemoral osteoarthritis). Osteoarthritis and Cartilage, 22(5), 631-638. https://doi.org/10.1016/j.joca.2014.02.008.

Smith, B. E., Moffatt, F., Hendrick, P., Bateman, M., Rathleff, M. S., Selfe, J., et al. (2018). The experience of living with patellofemoral pain - loss, confusion and fear-avoidance: A UK qualitative study. BMJ Open, 8(1). e018624 https://doi.org/ 10.1136/bmjopen-2017-018624.

Smith, B. E., Selfe, J., \& Rathleff, M. S. (2018). Incidence and prevalence of patellofemoral pain: A systematic review and meta-analysis. PLoS One, 13(1). https:// doi.org/10.1371/journal.pone.0190892.

Souza, T. A. (1997). In T. E. Hyde, \& M. S. Gengenbach (Eds.), Conservative management of sport injuries. Maryland: Williams \& Wilkins.

Wallace, D. a, Salem, G. J., Salinas, R., \& Powers, C. M. (2002). Patellofemoral joint kinetics while squatting with and without an external load. Journal of Orthopaedic \& Sports Physical Therapy, 32(4), 141-148.

Wyndow, N., Collins, N., Vicenzino, B., Tucker, K., \& Crossley, K. (2016). Is there a biomechanical link between patellofemoral pain and osteoarthritis? A narrative review. Sports Medicine, 46(12), 1797-1808. https://doi.org/10.1007/s40279016-0545-6. 